\title{
Switching Quantum Interference in Phenoxyquinone Single Molecule Junction with Light
}

\author{
Abdalghani Daaoub, Sara Sangtarash and Hatef Sadeghi * \\ Device Modelling Group, School of Engineering, University of Warwick, Coventry CV4 7AL, UK; \\ Abdalghani.Daaoub@warwick.ac.uk (A.D.); Sara.Sangtarash@warwick.ac.uk (S.S.) \\ * Correspondence: Hatef.Sadeghi@warwick.ac.uk
}

Received: 18 July 2020; Accepted: 3 August 2020; Published: 6 August 2020

\begin{abstract}
Quantum interference (QI) can lead to large variations in single molecule conductance. However, controlling QI using external stimuli is challenging. The molecular structure of phenoxyquinone can be tuned reversibly using light stimulus. In this paper, we show that this can be utilized to control QI in phenoxyquinone derivatives. Our calculations indicate that, as a result of such variation in molecular structure of phenoxyquinone, a crossover from destructive to constructive QI is induced. This leads to a significant variation in the single molecule conductance by a couple of orders of magnitude. This control of QI using light is a new paradigm in photosensitive single molecule switches and opens new avenues for future QI-based photoswitches.
\end{abstract}

Keywords: photochromic molecules; quantum interference; electrical conductance; molecular electronics; photoswitches

\section{Introduction}

Studying charge transport through molecular devices has attracted a growing interest in the last decade [1-3]. In particular, understanding the influence of light on properties of single molecular-scale devices can have applications on better characterization of the molecular junctions e.g., Raman spectroscopy or used as an alternative to a molecular scale field effect transistor [4-6]. In single molecule optoelectronics, one of the main motivations is to switch electrical conductance on and off using an external stimulus such as an external electric field [7,8], redox chemistry [9], or light $[4,5,10,11]$. In the latter, the electrical current is switched reversibly on and off in photochromic molecules in the presence of light or a change in its intensity. These can be mediated by electron transfers, photo-induced bond cleavages, pericyclic reactions, $E-Z$ isomerizations or intra-molecular hydrogen/group transfer [4,12-17]. In $E-Z$ isomerizations, the structure of molecule changes upon irradiation with light at specific wavelengths [18]. For example, the molecular structure of azobenzene [19] and stilbene [20] changes from trans to cis confirmation by ultraviolet light [10]. This leads to changes in the electrical conductance of the molecule between two electrodes. The conductance measurement of azobenzene between metallic electrodes shows that the single molecule conductance of cis confirmation is higher than trans confirmation by only a factor of two in agreement with predictions from theory (see Section A of the supporting information SI and Figures S1 and S2) [21,22]. This ratio is higher in self-assembled monolayers formed by azobenzene molecules because of changes in contacting modality and molecular film thickness [23,24]. However, these ratios are not sufficient to deliver high efficiency photoswitches.

Quantum interference (QI) can lead to a large variation (e.g., couple of orders of magnitude) in single molecule conductance as demonstrated in several molecular junctions [25]. For example, by simply changing the connection point to electrodes from para to meta in oligo(phenylene-ethynylenes) OPE3, the single molecule conductance changes by more than 30 times at room temperature due to 
a crossover from a destructive to constructive QI [26]. However, in-situ chemical modification of a molecule in the junction using external stimulus is challenging.

In what follows, our aim is to demonstrate that QI in a single molecule junction can be modulated from destructive to constructive in phenoxyquinone photosensitive molecules. Trans and cis configurations of phenoxyquinone are shown in Figure 1. These isomers are stable under different light stimuli [27]. In visible light, phenoxyquinone molecule takes trans configuration (Figure 1a) whereas under UV light it is changed to cis (Figure 1b) configuration [28]. The energy needed for this transition is c.a. $300 \mathrm{meV}$ [27]. This process is reversible and cis isomer can be transformed to trans isomer through irradiation or heating with visible light [29]. This makes phenoxyquinone derivatives attractive candidates for photoswitching. Here, we demonstrate for the first time that this light induced change in molecular structure controls room temperature QI in of phenoxyquinone molecules and a crossover from destructive QI (DQI) to constructive QI (CQI) takes place. This is significant because such crossover from CQI to DQI does not take place in other photochromic molecules such as azobenzene or dithienylethene leading to smaller on/off ratio. The crossover from CQI to DQI in phenoxyquinone leads to a couple of orders of magnitude increase in electrical current and a large on/off ratio of several orders of magnitude is accessible.
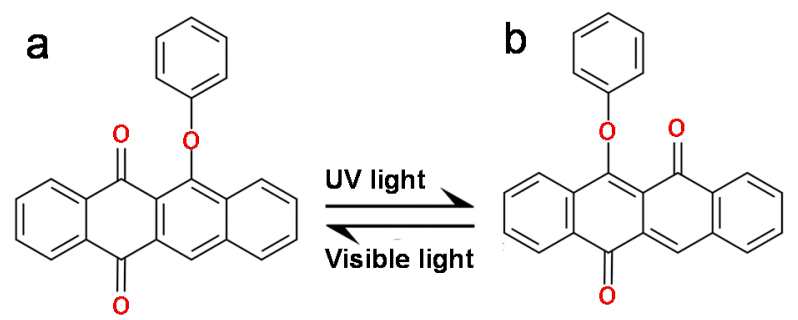

Figure 1. Chemical structures of (a) trans and (b) cis phenoxyquinone isomers. In visible light, phenoxyquinone molecule takes trans configuration, whereas, under UV light, it is changed to cis confirmation.

\section{Computational Methods}

The Hamiltonian of the structures described in this paper was obtained using DFT as described below or constructed from a simple tight-binding model with a single orbital per atom. The optimized geometry and ground-state Hamiltonian and overlap matrix elements of each structure were self-consistently obtained using the SIESTA [30] implementation of density functional theory DFT. SIESTA employs norm-conserving pseudopotentials to account for the core electrons and linear combinations of atomic orbitals to construct the valence states. The generalized gradient approximation (GGA) of the exchange and correlation functional is used with the Perdew-Burke-Ernzerhof parameterization, a double- $\zeta$ polarized basis set, and a real space grid defined with an equivalent energy cutoff of $250 \mathrm{Ry}$. The geometry optimization for each structure is performed for the forces smaller than $40 \mathrm{meV} / \AA$.

The mean-field Hamiltonian obtained from the converged DFT calculation or a simple tight-binding Hamiltonian was combined with Gollum [31,32] implementation of the non-equilibrium Green's function method to calculate the phase-coherent, elastic scattering properties of the each system consist of left (source) and right (drain) leads and the scattering region. The transmission coefficient $T(E)$ for electrons of energy $\mathrm{E}$ (passing from the source to the drain) is calculated via the relation $T(E)=$ $\operatorname{trace}\left(\Gamma_{R}(E) G^{R}(E) \Gamma_{L}(E) E^{R^{\dagger}}(E)\right)$. In this expression, $\Gamma_{L, R}(E)=i\left(\sum_{L, R}(E)-\sum_{L, R}^{+}(E)\right)$ describes the level broadening due to the coupling between left $(\mathrm{L})$ and right $(\mathrm{R})$ electrodes and the central scattering region, $\Sigma_{L, R}(E)$ are the retarded self-energies associated with this coupling, and $G_{R}=\left(E S-H-\Sigma_{L}-\Sigma_{R}\right)^{-1}$ is the retarded Green's function, where $H$ is the Hamiltonian and $S$ is overlap matrix. Using the obtained transmission coefficient $T(E)$, the electrical current I and conductance $\mathrm{G}$ could be calculated by the 
Landauer formula $I=\frac{e}{h} \int d E T(E)(f(e V / 2)-f(-e V / 2)) G=G_{0} \int d E T(E)\left(-\frac{\partial f}{\partial E}\right)$, where $G_{0}=2 \mathrm{e}^{2} / h$ is the conductance quantum, $V$ is bias voltage, and $f$ is Fermi distribution function. At low temperatures (e.g., $\mathrm{T}=0 \mathrm{~K}$ ), Landauer formula simplifies to $G=G_{0} T\left(E_{F}\right)$.

\section{Results}

We use density functional theory (DFT) to obtain ground state geometry of cis and trans phenoxyquinone isomers (see method section). Our calculation shows that the ground state energy of trans isomer is $310 \mathrm{meV}$ lower than cis isomer in agreement with reported experimental values [27]. The highest occupied molecular orbital (HOMO) and lowest unoccupied molecular orbital (LUMO) are shown in Figure S3 of the SI. Clearly, the HOMO-LUMO energy gap of trans-phenoxyquinone $(2.02 \mathrm{eV})$ is $0.5 \mathrm{eV}$ larger than cis-phenoxyquinone $(1.52 \mathrm{eV})$. To study electron transport properties of phenoxyquinone derivatives, we use two different anchor groups, thiol and direct $\mathrm{Au}-\mathrm{C}$ bond to attach molecules to gold electrodes (Figures 2 and 3). We then obtain the ground state mean field Hamiltonian from optimized geometry of each structure using DFT. The transmission probability [31] $T(E)$ of electrons with energy $E$ traversing through each junction is then calculated. The electrical conductance $G=G_{0} T\left(E_{F}\right)$ and current $I$ are then obtained from Landauer formula (see methods). Note that room temperature electrical conductance is obtained from thermal averaging of transmission function using Fermi distribution.

Figure $2 \mathrm{a}, \mathrm{b}$ show the molecular junctions formed by trans and cis phenoxyquinone molecules using thiol anchor, respectively. The electrical conductance of trans-phenoxyquinone is shown by a blue curve in Figure 2c. The dip in $G\left(E_{F}\right)$ is due to destructive quantum interference which leads to a low electrical conductance in trans isomer (dashed lines show the room temperature electrical conductance). In contrast, constructive interference through cis-phenoxyquinone leads to a higher conductance. At DFT Fermi energy $\left(E_{F}=0 \mathrm{eV}\right)$, the conductance is $8 \times 10^{-3} G_{0}$ for cis and $3 \times 10^{-5} G_{0}$ for trans isomers. Clearly, the conductance of cis-phenoxyquinone is at least two orders of magnitude higher than that of trans-phenoxyquinone not only at $E_{F}=0 \mathrm{eV}$, but also for whole energy range between HOMO and LUMO of trans-phenoxyquinone (Figure $2 \mathrm{c}$ ). Figure $2 \mathrm{~d}$ shows the room temperature electrical current through phenoxyquinone for different bias voltages between two electrodes (Figure 2a,b). The current is more than two orders of magnitude larger in cis isomer compared to that of trans isomer. The transition from low conductance trans isomer (off state) which can be induced by UV light to high conductance cis isomer (on state) leads to an on/off ratio of $>100$. This large variation in electrical conductance from cis to trans phenoxyquinone demonstrates the potential of these molecular junctions for photoswitching application.

To demonstrate that this large variation in electrical conductance is independent of the choice of the anchor group to electrodes, we calculate electron transport through phenoxyquinone derivatives using direct coupling to the gold electrodes (Au-C bond) as shown in Figure 3a,b. Figure $3 \mathrm{c}$ shows the conductance $G\left(E_{F}\right)$ of junctions of Figure 3a,b. Clearly, the electron transmission through trans-phenoxyquinone is governed by DQI leading to a low electrical conductance, whereas electrons transmit through cis-phenoxyquinone junction constructively (high conductance). The conductance values are $1 \times 10^{-2} G_{0}$ and $2 \times 10^{-4} G_{0}$ for cis and trans isomers, respectively, at $E_{F}=0 \mathrm{eV}$. The electrical conductance through cis-phenoxyquinone is $2-5$ orders of magnitude higher than trans-phenoxyquinone for a wide energy range in the vicinity of $E_{F}=0 \mathrm{eV}$, meaning that, regardless of the Fermi energy of electrodes, the electrical conductance of cis isomer is much higher than that of trans isomer. Figure $3 \mathrm{~d}$ shows the electrical current for junctions of Figure $3 \mathrm{a}, \mathrm{b}$ for different bias voltages at room temperature. Clearly, a large on/off ratio of $>1000$ is accessible at $V_{b}>0.45 \mathrm{~V}$. This is higher than the on/off ratio of dithienylethene, azobenzene, and stilbene photochromic molecules studied between gold or graphene electrodes $[10,11,15,33]$ and is a consequence of crossover from DQI to CQI in phenoxyquinone molecules. 

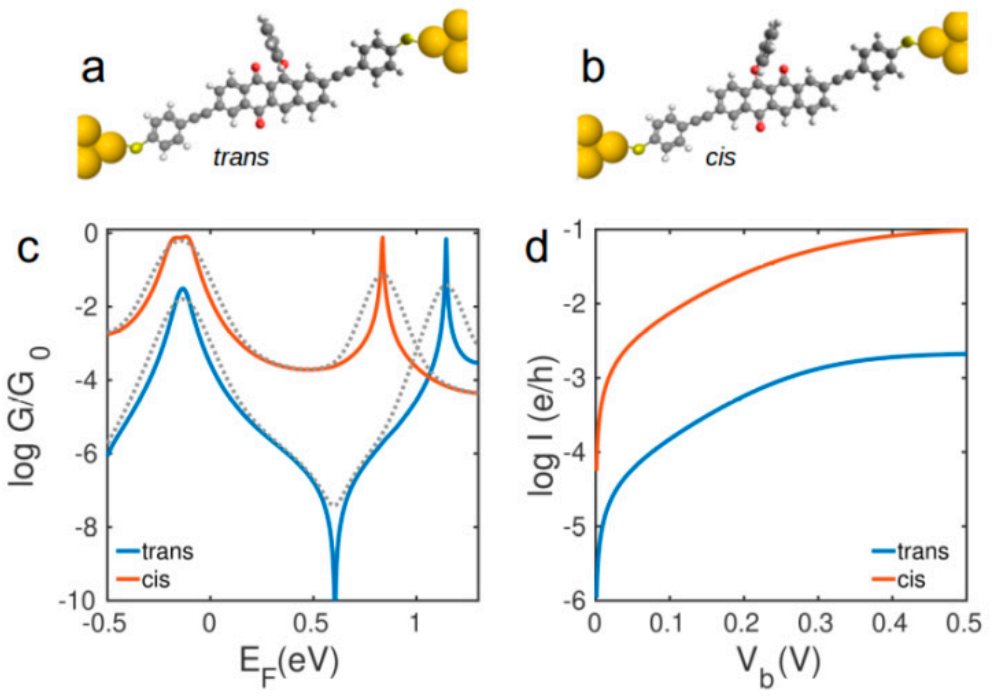

Figure 2. Molecular structures of (a) trans and (b) cis isomers of phenoxyquinone with thiol anchor connected to gold electrodes, (c) low temperature conductance and (d) room temperature current for $(\mathbf{a}, \mathbf{b})$. Dashed lines in (c) show the corresponding room temperature conductances.
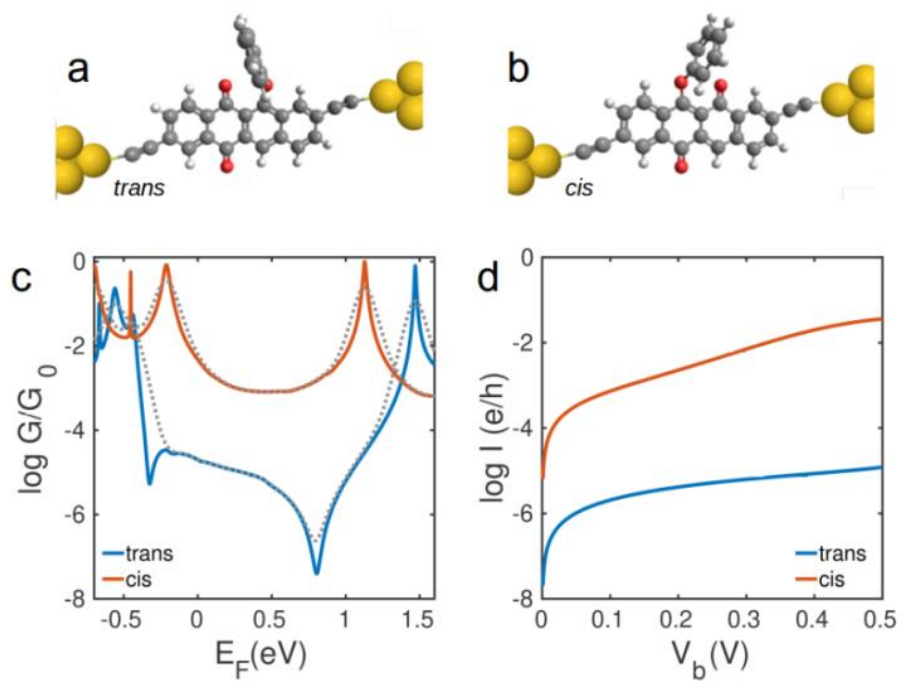

Figure 3. Molecular structures of (a) trans and (b) cis isomers of phenoxyquinone with acetylene linker and direct Au-C connection to gold electrodes; (c) low temperature conductance and (d) room temperature current for $(\mathbf{a}, \mathbf{b})$. Dashed lines in (c) show the corresponding room temperature conductances.

This large variation in the electrical conductance is due to a crossover from conjugated cisphenoxyquinone to cross conjugated trans-phenoxyquinone. This can be understood using curly arrows rules [34]. Arrow-pushing pathways are 'dead ends' in trans configuration (Figure 4a), whereas there is a 'pathway' from one electrode to the other in cis configuration (Figure $4 \mathrm{~b}$ ). This means that, from curly arrows rules, a DQI (CQI) is expected for cis (trans) isomer in agreement with DFT results in Figures 2 and 3. The oxygen atom in carbonyl group $(C=O)$ acts like scissors which weaken the electronic coupling between the neighbouring carbon atom (shown by green dots in Figure 4c,d) with its other neighbouring atoms (shown with blue coupling in Figure $4 \mathrm{c}, \mathrm{d}$ ). The oxygen atom in carbonyl group also changes on-site energy of neighbouring carbon atoms by modifying the charge on the carbon atom. Using this picture, we build a simple single orbital per atom tight binding model as shown in Figure 4 (see methods for details). We then calculate transmission coefficient $T(E)$ between site $m$ and $n$ (Figure 4c,d) for both trans and cis isomers as shown in Figure 4e. We obtain DQI in trans 
isomer in contrast to CQI in cis isomer (Figure 4e) in qualitative agreement with our material specific DFT result (Figures 2 and 3).
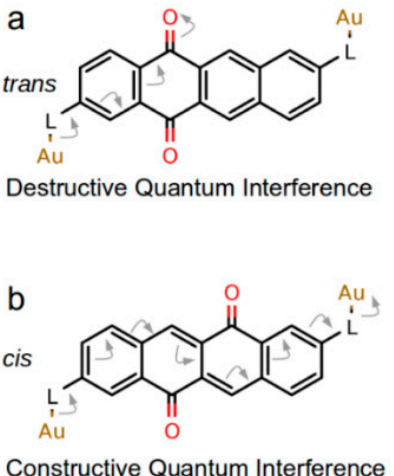

C

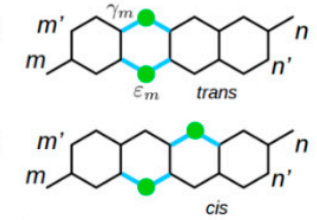

e

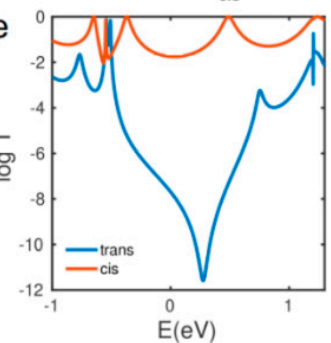

Figure 4. QI based on curly arrows rules and tight binding model. Arrow-pushing pathways for (a) cis and (b) trans phenoxyquinone isomers. (c,d) simplified tight binding picture and (e) electron transmission coefficient curves for trans and cis isomers as a function of electron energy for tight-binding model of site energy $\varepsilon_{0}=0$ and nearest-neighbor couplings $\gamma=-1$. On-site energy at red sites (Figure 4) is $\varepsilon_{m}=-0.55 \gamma$ and $\gamma_{m}=-0.15$.

In order to demonstrate the effect of connection point to the electrodes on QI through trans and cis configurations of phenoxyquinone, we also calculate transmission coefficient between $\left(m, n^{\prime}\right),\left(m^{\prime}, n\right)$ and $\left(m^{\prime}, n^{\prime}\right)$ connectivities (Figure $\left.4 \mathrm{c}, \mathrm{d}\right)$ using material specific Hamiltonians obtained from DFT. We obtain constructive interference for both cis and trans isomers when they connected to the electrodes from $\left(m, n^{\prime}\right)$ and $\left(m^{\prime}, n\right)$ connectivities (Figure S5a-f of the Supplementary Materials), whereas quantum interference is destructive for both cis and trans isomers through $\left(m^{\prime}, n^{\prime}\right)$ connectivity (Figure S5g-i of the Supplementary Materials). This shows that the manifestation of light induced QI depends on the connection point of phenoxyquinone core to the electrodes. This is also in agreement with our simple tight-binding model (Figures S5j-1 and S7j-1 of the Supplementary Materials). Using different anchor groups or electrode configuration does not change the result as shown in Figures S6 and S7 of the Supplementary Materials.

\section{Conclusions}

In summary, photochromic molecules change their conformation when exposed to ultraviolet or visible light irradiation. In this paper, we demonstrated that such conformational change in phenoxyquinone derivatives can lead to a significant variation in their single molecule conductance by a couple of orders of magnitude. We attributed this to a reversible crossover from destructive interference in trans isomer to constructive interference in cis isomer, which leads to a large variation in current (>1000 times) at room temperature.

Supplementary Materials: The following are available online at http://www.mdpi.com/2079-4991/10/8/1544/s1, Figure S1. Frontier molecular orbitals of azobenzene. Figure S2. Transport through of azobenzene derivatives. Figure S3. Frontier molecular orbitals of phenoxyquinone. Figure S4. Energy and isosurfaces of phenoxyquinone molecular orbitals for trans and cis isomers. Figure S5. Molecular structure of the junctions formed phenoxyquinone isomers and electron transport through the junctions for different connectivities to electrodes. Figure S6. Molecular structure of the junctions formed phenoxyquinone isomers and electron transport through the junctions using different anchor / electrode configuration. Figure S7. Molecular structure of the junctions formed phenoxyquinone isomers and electron transport through the junctions for different connectivities to electrodes using direct Au-C.

Author Contributions: Conceptualization, H.S.; Calculations, analysis and writing, A.D., S.S., and H.S., supervision, H.S. All authors have read and agreed to the published version of the manuscript. 
Funding: This research was funded by the UKRI for Future Leaders Fellowship, Grant No. MR/S015329/2 (H.S.) and the Leverhulme Trust for Early Career Fellowship Grant No. ECF-2018- 375 (S.S.).

Conflicts of Interest: The authors declare no conflict of interest.

\section{References}

1. Seong, H.C.; Kim, B.; Frisbie, C.D. Electrical resistance of long conjugated molecular wires. Science 2008, 320, 1482-1486. [CrossRef]

2. Reed, M.A.; Zhou, C.; Muller, C.J.; Burgin, T.P.; Tour, J.M. Conductance of a molecular junction. Science 1997, 278, 252-254. [CrossRef]

3. Tao, N.J. Electron transport in molecular junctions. Nat. Nanotechnol. 2006, 1, 173-181. [CrossRef]

4. Russew, M.-M.; Hecht, S. Photoswitches: From Molecules to Materials. Adv. Mater. 2010, 22, 3348-3360. [CrossRef]

5. Goulet-Hanssens, A.; Eisenreich, F.; Hecht, S. Enlightening Materials with Photoswitches. Adv. Mater. 2020, 1905966. [CrossRef]

6. Chikkaraddy, R.; De Nijs, B.; Benz, F.; Barrow, S.J.; Scherman, O.A.; Rosta, E.; Demetriadou, A.; Fox, P.; Hess, O.; Baumberg, J.J. Single-molecule strong coupling at room temperature in plasmonic nanocavities. Nature 2016, 535, 127-130. [CrossRef]

7. Harzmann, G.D.; Frisenda, R.; Van Der Zant, H.S.J.; Mayor, M. Single-Molecule Spin Switch Based on Voltage-Triggered Distortion of the Coordination Sphere. Angew. Chem. Int. Ed. 2015, 54, 13425-13430. [CrossRef]

8. Zhang, J.L.; Zhong, J.Q.; Lin, J.D.; Hu, W.P.; Wu, K.; Xu, G.Q.; Wee, A.T.S.; Chen, W. Towards single molecule switches. Chem. Soc. Rev. 2015, 44, 2998-3022. [CrossRef]

9. Canary, J.W. Redox-triggered chiroptical molecular switches. Chem. Soc. Rev. 2009, 38, 747. [CrossRef]

10. Zhang, X.; Hou, L.; Samorì, P. Coupling carbon nanomaterials with photochromic molecules for the generation of optically responsive materials. Nat. Commun. 2016, 7, 11118. [CrossRef]

11. Huang, X.; Li, T. Recent progress in the development of molecular-scale electronics based on photoswitchable molecules. J. Mater. Chem. C 2020, 8, 821-848. [CrossRef]

12. Bouas-Laurent, H.; Dürr, H. Organic photochromism (IUPAC Technical Report). Pure Appl. Chem. 2001, 73, 639-665. [CrossRef]

13. Jia, C.; Wang, J.; Yao, C.; Cao, Y.; Zhong, Y.; Liu, Z.; Liu, Z.; Guo, X. Conductance switching and mechanisms in single-molecule junctions. Angew. Chem. Int. Ed. 2013, 52, 8666-8670. [CrossRef]

14. Kim, Y.; Hellmuth, T.J.; Sysoiev, D.; Pauly, F.; Pietsch, T.; Wolf, J.; Erbe, A.; Huhn, T.; Groth, U.; Steiner, U.E.; et al. Charge transport characteristics of diarylethene photoswitching single-molecule junctions. Nano Lett. 2012. [CrossRef]

15. Dulić, D.; van der Molen, S.J.; Kudernac, T.; Jonkman, H.T.; de Jong, J.J.D.; Bowden, T.N.; van Esch, J.; Feringa, B.L.; van Wees, B.J. One-way optoelectronic switching of photochromic molecules on gold. Phys. Rev. Lett. 2003, 91, 207402. [CrossRef]

16. Marquardt, C.W.; Grunder, S.; Błaszczyk, A.; Dehm, S.; Hennrich, F.; Löhneysen, H.V.; Mayor, M.; Krupke, R. Electroluminescence from a single nanotube-molecule-nanotube junction. Nat. Nanotechnol. 2010, 5, 863-867. [CrossRef]

17. Roldan, D.; Kaliginedi, V.; Cobo, S.; Kolivoska, V.; Bucher, C.; Hong, W.; Royal, G.; Wandlowski, T. Charge transport in photoswitchable dimethyldihydropyrene-type single-molecule junctions. J. Am. Chem. Soc. 2013, 135, 5974-5977. [CrossRef]

18. Purkait, M.K.; Sinha, M.K.; Mondal, P.; Singh, R. Photoresponsive Membranes. Interface Sci. Technol. 2018, 25, 115-144. [CrossRef]

19. Rau, H. Photoisomerization of Azobenzenes. In Photoreactive Organic Thin Films; Elsevier: Amsterdam, The Netherlands, 2002; pp. 3-47.

20. Martin, S.; Haiss, W.; Higgins, S.J.; Nichols, R.J. The Impact of E-Z Photo-Isomerization on Single Molecular Conductance. Nano Lett. 2010, 10, 2019-2023. [CrossRef]

21. Qin, M.; Huang, Y.; Li, F.; Song, Y. Photochromic sensors: A versatile approach for recognition and discrimination. J. Mater. Chem. C 2015, 3, 9265-9275. [CrossRef] 
22. Osella, S.; Samorì, P.; Cornil, J. Photoswitching Azobenzene Derivatives in Single Molecule Junctions: A Theoretical Insight into the I/V Characteristics. J. Phys. Chem. C 2014, 118, 18721-18729. [CrossRef]

23. Mativetsky, J.M.; Pace, G.; Elbing, M.; Rampi, M.A.; Mayor, M.; Samorì, P. Azobenzenes as Light-Controlled Molecular Electronic Switches in Nanoscale Metal-Molecule-Metal Junctions. J. Am. Chem. Soc. 2008, 130, 9192-9193.

24. Kumar, A.S.; Ye, T.; Takami, T.; Yu, B.C.; Flatt, A.K.; Tour, J.M.; Weiss, P.S. Reversible photo-switching of single azobenzene molecules in controlled nanoscale environments. Nano Lett. 2008, 8, 1644-1648. [CrossRef]

25. Xiang, D.; Wang, X.; Jia, C.; Lee, T.; Guo, X. Molecular-Scale Electronics: From Concept to Function. Chem. Rev. 2016, 116, 4318-4440. [CrossRef]

26. Liu, X.; Sangtarash, S.; Reber, D.; Zhang, D.; Sadeghi, H.; Shi, J.; Xiao, Z.Y.; Hong, W.; Lambert, C.J.; Liu, S.X. Gating of Quantum Interference in Molecular Junctions by Heteroatom Substitution. Angew. Chem. Int. Ed. 2017, 56, 173-176. [CrossRef]

27. Park, I.S.; Heo, E.J.; Kim, J.M. A photochromic phenoxyquinone based cyanide ion sensor. Tetrahedron Lett. 2011, 52, 2454-2457. [CrossRef]

28. Pace, G.; Ferri, V.; Grave, C.; Elbing, M.; von Hanisch, C.; Zharnikov, M.; Mayor, M.; Rampi, M.A.; Samori, P. Cooperative light-induced molecular movements of highly ordered azobenzene self-assembled monolayers. Proc. Natl. Acad. Sci. USA 2007, 104, 9937-9942. [CrossRef]

29. Moresco, F.; Meyer, G.; Rieder, K.-H.; Tang, H.; Gourdon, A.; Joachim, C. Conformational Changes of Single Molecules Induced by Scanning Tunneling Microscopy Manipulation: A Route to Molecular Switching. Phys. Rev. Lett. 2001, 86, 672-675. [CrossRef]

30. Soler, J.M.; Artacho, E.; Gale, J.D.; García, A.; Junquera, J.; Ordejón, P.; Sánchez-Portal, D. The SIESTA method for ab initio order- N materials simulation. J. Phys. Condens. Matter. 2002, 14, 2745-2779. [CrossRef]

31. Sadeghi, H. Theory of electron, phonon and spin transport in nanoscale quantum devices. Nanotechnology 2018, 29, 373001. [CrossRef]

32. Ferrer, J.; Lambert, C.J.; García-Suárez, V.M.; Manrique, D.Z.; Visontai, D.; Oroszlany, L.; Rodríguez-Ferradás, R.; Grace, I.; Bailey, S.W.D.; Gillemot, K.; et al. GOLLUM: A next-generation simulation tool for electron, thermal and spin transport. New J. Phys. 2014, 16, 093029. [CrossRef]

33. Jia, C.; Migliore, A.; Xin, N.; Huang, S.; Wang, J.; Yang, Q.; Wang, S.; Chen, H.; Wang, D.; Feng, B.; et al. Covalently bonded single-molecule junctions with stable and reversible photoswitched conductivity. Science 2016, 352, 1443-1445. [CrossRef]

34. Kermack, W.O.; Robinson, R. LI.-An explanation of the property of induced polarity of atoms and an interpretation of the theory of partial valencies on an electronic basis. J. Chem. Soc. Trans. 1922, 121, 427-440. [CrossRef]

(C) 2020 by the authors. Licensee MDPI, Basel, Switzerland. This article is an open access article distributed under the terms and conditions of the Creative Commons Attribution (CC BY) license (http://creativecommons.org/licenses/by/4.0/). 